\title{
Fibers of Glass
}

Though glassmakers have known since ancient times that glass could be drawn out into fine filaments, the enormous number of applications for glass fibers was not realized until early this century. Glass fibers are now used in long single strands for fiber-optic communications, in short tangled mats for insulation, and mixed with other structural materials to provide extra strength.

Glass fibers were used as early as 1500 B.C. In the Egyptian XVIIIth Dynasty, glassmakers created small vases for cosmetics by attaching either a small clay form or a bag filled with sand to a stick. Rotating this form in molten glass would result in a small vase of the desired shape. Coarse fibers of colored glass were then heated and pressed into the walls of the vessels to make decorative patterns. Similar decorative fibers were wound around glass dishes, cups, and bottles in the Rhineland of the late Roman Empire.

In the 18th Century, glassmakers made finer fibers by drawing a thread from a heat-softened glass rod onto a spinning wheel. As a novelty, Carlo Reva of Venice wove some of this fine "spun glass" into a sample of cloth. In 1713, Rene Reaumur submitted Reva's glass cloth to the Paris Academy of Science for inspection. An entire garment made from woven glass was given to a Spanish princess in the 1860s, but the fiber was too coarse to allow the garment to be folded.

In 1908, G. von Pazsiczky developed a method to draw several fibers from holes in the bottom of a refractory glass-melting chamber. In 1911, E. Pick received a patent for his technique of using centrifugal force to draw fibers through tiny holes in a drum. During the first World War, Germany used these methods to make fiberglass as a substitute for asbestos.

In the 1920s, W. Schuller found a new way to make fiberglass out of glass rods, drawing strands from a hundred such rods that had been gas or electrically heated, then winding the strands onto a single drum.

In the 1930s, the Owens-nllinois Glass Company and the Corning Glass Works combined their separate fiberglass research centers to form Owens-Corning Fiberglas. G. Slayter, J.H. Thomas, and others working for Owens-Corning developed mass-production methods that allowed the industrialization of fiberglass manufacture from 1936-1950.

To draw out a continuous, single glass filament, such as for making optical fibers and glass yarn, molten glass is fed through precious-metal spinnerets with very accurate holes in their ends. About 400 such spinnerets can be used in any single machine, and thin glass fibers can be drawn off at a rate of several miles per minute. One piece of feed glass $1.9 \mathrm{~cm}$ in diameter could yield about $156 \mathrm{~km}$ of filament from this process.

On the other hand, the production of matted glass wool demands a dense cluster of many short, fine fibers. In Britain in 1929, the Hager brothers and F. Rosengarth succeeded in developing a new process for making glass wool, announcing it before the American investigators Harford and
Stafford could complete their similar work. In the Hager process, a stream of molten glass falls on a fire-clay disk with radial serrations that rotate rapidly about a vertical axis. This disk threw off short, thin fibers tangentially...along with large blobs of glass.

The Cie de St. Gobain and OwensCorning both improved the centrifugal process in the 1950s, this time replacing the refractory disk with a metal spinner pierced with thousands of tiny holes. The vertical cylinder spins, drawing out a fiber from each hole through centrifugal force; the fibers are deflected downward and further attenuated by a blowing ring. The fibers are bonded with a resin, collected on a conveyor belt, then subjected to another

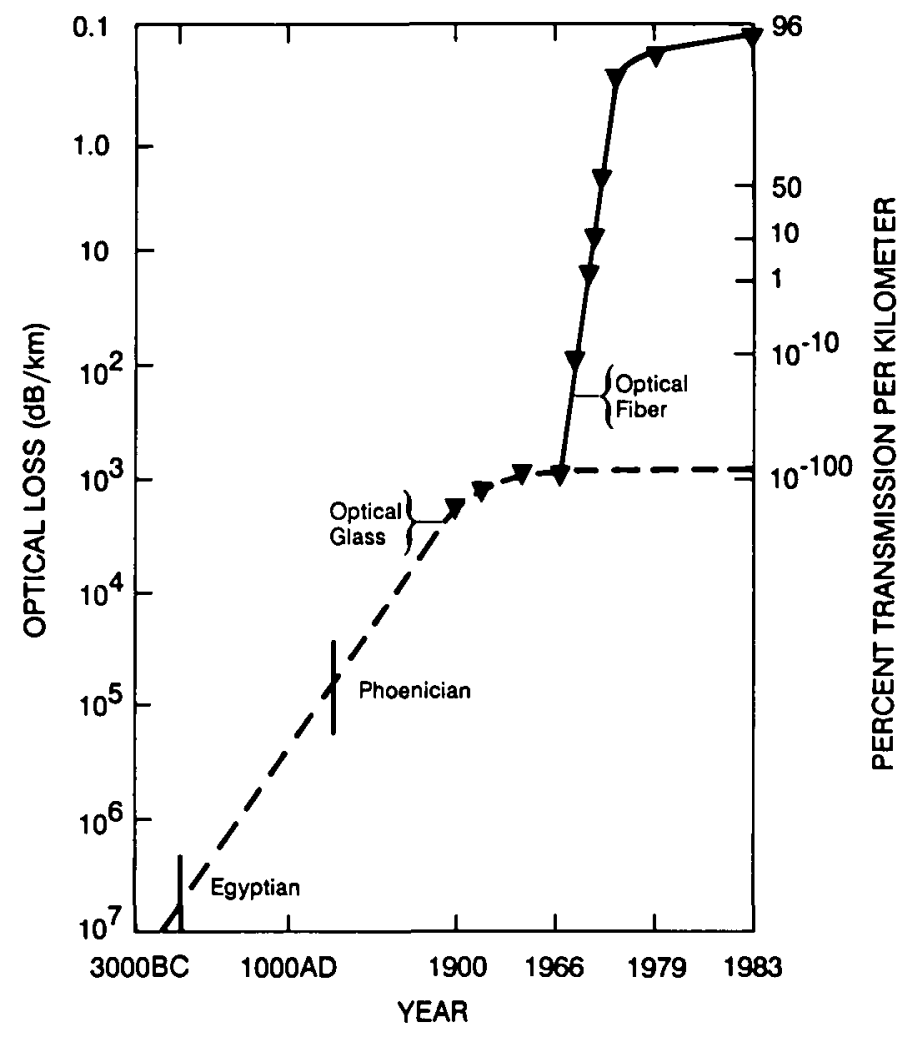

Historical improvement in glass transparency. Scientists and technicians improved the transparency of silica glass slowly over the centuries from 3000 B.C. to 1966, when work on optical fibers was begun in earnest. Today, these fibers are some 100 orders of magnitude more transparent than they were in 1966 . A single glass fiber $0.01 \mathrm{~mm}$ in diameter can transmit thousands of telephone conversations-many more by far than can be sent over a conventional cable. Figure and text from Materials Science and Engineering for the 1990s, National Research Council (National Academy Press, Washington, DC, 1989) p. 23-24, with permission. 
blower that tangles the fibers into a random mat.

Fibers formed with this production technique range from 6 to $12 \mu \mathrm{m}$ in diameter and have a good degree of uniformity. The natural density of glass wool made this way is about $24 \mathrm{~kg} / \mathrm{m}^{3}$ and can be used at temperatures up to about $500^{\circ} \mathrm{C}$.

Each strand of fiberglass, though thinner than a human hair, is still a solid glass rod, and so retains the bulk material properties of its component glass. In general, fiberglass is heat resistant and noncombustible (making it a good substitute for asbestos). Fiberglass can't rot or decay, nor does the glass itself absorb moisture. It doesn't conduct electricity, and it is resistant to ordinary weathering, acids, and corrosive vapors. Glass fibers are very strong, but have little ductility; the thinner the fiber, however, the more it can bend without breaking. Single glass fibers can be woven into yarns, cords, braids, cloths, or tapes; glass wool can be used as excellent thermal and sound insulators. Most glass fibers are colorless, but the strands can be coated with organic resins containing dyes or pigments.

Glass fibers were used as early as 1500 B.C. be stronger per unit weight than other common structural materials such as steel, titanium, magnesium, or aluminum. The first commercial use of fiberglassreinforced plastics was in 1942 in the manufacture of housings for aircraft radar systems (radomes) and in other structural parts of aircraft and naval combat vessels. Fiberglass-reinforced plastic boat hulls were made in the United States in 1946; seaplane floats were made in Britain in 1947.

Until the late 1940s, the combined cost of the resin and the fiberglass precluded major commercial development of composite materials. In 1947, it was found that the reinforcing fibers need not be so fine; coarser fiber mats could be made more cheaply, which opened up new techniques such as diamond-pattern mat and swirled mat fabrics and plastics.

In 1951, I.A. Rubinsky in the United States investigated the use of fiberglass as reinforcement for concrete, but he hypothesized from his initial tests that fiberglass would not replace steel as ordinary reinforcement because of the low modulus of elasticity of glass. However, in 1957, K.L. and U.L. Biryukovich at the laboratories of Building Construction of the Kiev Polytechnic Institute of Lenin used continuous three-ply glass fibers as well as chopped strands. They found that though the fiberglass required some protective coating against the alkali in the cement, its use as construction reinforcement was quite feasible. In the intervening years, the quality of commercially available fiberglass has greatly improved, increasing its ultimate tensile strength and its Young's modulus, making fiberglass-reinforced concrete an important building material.

New uses for fiberglass continue to be discovered, and the fiberglass industry has prospered not only in the United States and Britain, but in at least 30 other countries as well, including Australia, South Africa, Brazil, Argentina, India, Japan, countries in Southeast Asia, the U.S.S.R., and China.

A new and dramatic application for glass fibers is in the transmission of information-made possible through advances in laser technology and electronic signal detection and processing equipment, but mostly through major advances in developing ultrapure, ultratransparent glass materials. Glass fibers used for communication must have low transmittance loss at specific wavelengths and high fiber strengths to maintain integrity over transmission paths of many kilometers. Much progress is being made in ensuring the purity of the glass used in optical fibers, as well as precision in the dimensions of the fibers themselves. New formulations of glass are also being developed that address the changing material requirements for fiber-optic communication.

KEVIN J. ANDERSON
Fibers made from specific types of glass are used for particular applications. Lowalkali lime-alumina borosilicate fibers, which have excellent dielectric and weathering properties, are used for general and electrical textiles and for reinforcing plastics, papers, rubbers, and gypsum. Inexpensive soda-lime fiberglass is used for air and liquid filters. Soda-lime borosilicate fibers, with their high acid resistance, are used for corrosion protection and as mats for storage-battery retainers, as well as acid filter cloths and anode bags. The excellent weathering properties of lime-free soda borosilicate glass fibers makes them ideal for high-efficiency filter papers and for lightweight thermal and acoustical insulation material. Finally, high-lead silicate fiberglass, with its special $x$-ray opacity, is used for surgical pad strands and for $x$-ray protection aprons.

When researchers discovered that they could mix glass fibers with asbestos, mica, silicones, and thermosetting resins, new classes of materials became available to them. Some laminates made of parallel glass fibers in polyester resin were found to

\section{Find out what you can do to help science education...}

Come to a special networking session for 1990 MRS Fall Meeting attendees, Thursday, November 29 at 7:30 p.m. in the Staffordshire Room, Westin Hotel. Get together with your colleagues and find out how you can create a greater positive contact between the scientific community and students at the elementary, middle, and high school levels.

If you're interested in helping science education but can't come to this session, contact Kenneth $\mathrm{H}$. Eckelmeyer, Sandia National Laboratories, Organization 30, P.O. Box 5800, Albuquerque, NM 87185; telephone (505) 845-8680; FAX (505) 846-4168. 